\title{
Biometria e Potencial Germinativo em Sementes de Peltophorum dubium (Sprengel)
}

\section{Taubert - Fabaceae}

Biometry and Germinative Potential in Seeds of Peltophorum dubium (Sprengel) Taubert -

Fabaceae

Biometría y potencial germinativo en semillas de Peltophorum dubium (Sprengel) Taubert Fabaceae

\author{
Daniela Fialho Duarte \\ ORCID: https://orcid.org/0000-0002-7009-4810 \\ Universidade Estadual de Mato Grosso do Sul, Brasil \\ E-mail: danielafialhoduarte@gmail.com \\ Felipe Brancalion Giacomelli \\ ORCID: https://orcid.org/0000-0001-7538-3630 \\ Universidade Estadual de Mato Grosso do Sul, Brasil \\ E-mail: felipe.brancalion@yahoo.com.br \\ Simone Yasuda Fernandes \\ ORCID: https://orcid.org/0000-0003-1418-1394 \\ Universidade Estadual de Mato Grosso do Sul, Brasil \\ E-mail: simoneyasuda@hotmail.com \\ Bianca de Oliveira Souza \\ ORCID: https://orcid.org/0000-0002-8485-0656 \\ Universidade Estadual de Mato Grosso do Sul, Brasil \\ E-mail: bianca.033@hotmail.com \\ Gláucia Almeida de Morais \\ ORCID: https://orcid.org/0000-0002-6498-8164 \\ Universidade Estadual de Mato Grosso do Sul, Brasil \\ E-mail: gamorais@uems.br
}

\begin{abstract}
Resumo
A crescente busca por sementes de alta qualidade para reflorestamento tem aumentado o interesse pelos conhecimentos relacionados à biologia de sementes como as características biométricas, com intuito de tornar mais viável à produção de mudas de algumas espécies. Assim, objetivou-se no presente estudo analisar a biometria de sementes de diferentes matrizes de Peltophorum dubium (Spreng) Taubert, e relacioná-la ao potencial germinativo e à intensidade de dormência em diferentes tamanhos de sementes. As sementes foram coletadas em 12 matrizes e avaliadas quanto ao comprimento, largura, espessura e massa. Os dados obtidos foram submetidos a testes para a comparação das matrizes, verificação de relação entre as variáveis e para definição de categorias de sementes com base nas classes de massa (pequenas, médias e grandes). O potencial germinativo de cada matriz e das três categorias de sementes foi avaliado comparando sementes intactas e sementes submetidas à quebra de dormência em ácido sulfúrico. Os dados de germinação foram utilizados para os cálculos de porcentagem, frequência relativa, índice de velocidade e tempo médio. As matrizes e as categorias foram comparadas estatisticamente por análise de variância. A correlação dos dados biométricos das sementes mostrou-se positivamente significativa para todas as comparações, com associações mais fortes para as associações com massa, variável utilizada para a definição das categorias de tamanho. As sementes escarificadas obtiveram, em média, $90 \%$ de germinação enquanto o controle $4 \%$. As sementes médias e grandes apresentaram maior desempenho germinativo, sem influência do regime de luz (luz contínua e fotoperíodo de 12 horas). Concluímos neste estudo que sementes de Peltophorum dubium se assemelham biometricamente a lotes provenientes de regiões geograficamente mais próximas e que existe variabilidade entre matrizes, sendo este um fator importante para a manutenção das populações. Além disso, observamos também que a variável massa é um parâmetro adequado para a seleção de sementes com maior potencial germinativo.
\end{abstract}

Palavras-chave: Canafístula; Matriz; Categoria; Germinação; Superação de dormência.

\section{Abstract}

The growing search for high quality seeds for reforestation has increased the interest in knowledge related to seed biology such as biometric characteristics, in order to make the production of seedlings of some species more viable. Thus, the aim of this study was to analyze the biometry of seeds from different matrices of Peltophorum dubium (Spreng) Taubert, and relate it to germination potential and dormancy intensity in different seed sizes. Seeds were 
collected in 12 matrices and evaluated for length, width, thickness and mass. The data obtained were subjected to tests to compare the matrices, verify the relationship between the variables and to define seed categories based on mass classes (small, medium and large). The germination potential of each matrix and of the three seed categories was evaluated comparing intact seeds and seeds submitted to dormancy breaking in sulfuric acid. Germination data were used to calculate percentage, relative frequency, speed index and mean time. The matrices and categories were compared statistically by analysis of variance. The correlation of biometric data of the seeds was positively significant for all comparisons, with stronger associations for associations with mass, a variable used to define size categories. The scarified seeds obtained, on average, $90 \%$ germination while the control $4 \%$. Medium and large seeds showed better germination performance, without influence of the light regime (continuous light and 12-hour photoperiod). We conclude in this study that Peltophorum dubium seeds biometrically resemble lots from geographically closer regions and that there is variability between matrices, which is an important factor for population maintenance. Furthermore, we also observed that the mass variable is an adequate parameter for the selection of seeds with greater germination potential.

Keywords: Canafistula; Headquarters; Category; Germination; Overcoming dorman.

\begin{abstract}
Resumen
La creciente búsqueda de semillas de alta calidad para la reforestación ha incrementado el interés en conocimientos relacionados con la biología de las semillas como las características biométricas, con el fin de hacer la producción de plántulas de algunas especies más viables. Así, el objetivo de este estudio fue analizar la biometría de semillas de diferentes matrices de Peltophorum dubium (Spreng) Taubert, y reportarlo a potencial germinativo e intensidad de latencia en diferentes tamaños de semilla. Las semillas se recolectaron en 12 matrices y se evaluaron en cuanto a longitud, ancho, grosor y masa. Los datos obtenidos fueron sometidos a pruebas para comparar las matrices, verificar la relación entre las variables y definir categorías de semillas en base a clases de masa (pequeña, mediana y grande). El potencial de germinación de cada matriz y de las tres categorías de semillas se evaluó comparando semillas intactas y semillas sometidas a ruptura de la latencia en ácido sulfúrico. Los datos de germinación se utilizaron para calcular el porcentaje, la frecuencia relativa, el índice de velocidad y el tiempo medio. Las matrices y categorías se compararon estadísticamente mediante análisis de varianza. La correlación de los datos biométricos de las semillas fue positivamente significativa para todas las comparaciones, con asociaciones más fuertes de asociaciones con la masa, una variable utilizada para definir las categorías de tamaño. Las semillas escarificadas obtuvieron, en promedio, $90 \%$ de germinación mientras que el control 4\%. Semillas medianas y grandes mostraron mejor desempeño germinativo, sin influencia del régimen de luz (luz continua y fotoperiodo de 12 horas). Concluimos en este estudio que las semillas de Peltophorum dubium se asemejan biométricamente a lotes de regiones geográficamente más cercanas y que existe variabilidad entre matrices, lo cual es un factor importante para el mantenimiento de la población. Además, también observamos que la variable masa es un parámetro adecuado para la selección de semillas con mayor potencial germinativo.
\end{abstract}

Palabras clave: Canafistula; Sede; Categoría; Germinación; Superando dormán.

\title{
1. Introdução
}

Peltophorum dubium (Sprengel) Taub., conhecida popularmente como canafístula, pertence à família Fabaceae Caesalpinioideae e é comum em florestas latifoliadas semidecíduas da bacia do rio Paraná. É caracterizada por ser uma planta rústica e decídua, que floresce nos meses de dezembro a fevereiro, com maturação de seus frutos observada entre março e abril. Apresenta crescimento rápido, sendo considerada ótima para reflorestamentos mistos de áreas degradadas com preferência por solos argilosos úmidos e profundos de beira de rio (Lorenzi, 2008). Embora seja uma espécie indicada para práticas de restauração, a sua utilização é dificultada pelo conhecimento limitado da biologia básica da espécie, especialmente quando se trata dos condicionantes ambientais para a germinação (Ermínio et al., 2016).

Para o uso eficiente desta e outras espécies florestais em projetos de reflorestamento, a busca por sementes de alta qualidade tem aumentado, o que também torna necessário que os métodos de avaliação dessa qualidade sejam eficazes (Santos, 2007). Desta forma, estudos que visam obter um acervo de informações que auxiliem na compreensão das respostas germinativas das espécies, como, a biometria e a dormência de sementes se tornam essenciais.

A biometria da semente está relacionada às características e ao estabelecimento de plântulas (Fenner, 1993), trazendo dados úteis para identificar a variabilidade e os fatores ambientais (Gusmão et al., 2006). Os dados biométricos de frutos e sementes também contribuem para estudos de diferenciação de espécies (Cruz et al., 2001; Fontenele et al., 2007), classificação de grupos ecológicos e outros aspectos que podem contribuir para programas de melhoramento genético de 
sementes (Fontenele et al., 2007). Durante a maturação, as sementes crescem em tamanho até atingirem o valor característico para a espécie (Carvalho \& Nakagawa, 2000). Porém, dentro da mesma espécie existem variações individuais que se devem à influência ambiental durante o desenvolvimento das sementes e da variabilidade genética entre as matrizes. Desta forma, o tamanho das sementes pode variar entre as árvores matrizes da mesma espécie ou na mesma planta (Santos et al., 2009).

Já a dormência de sementes é caracterizada quando são reconhecidos em uma semente um ou mais fatores que bloqueiam ou inibem sua germinação, mesmo quando as sementes são expostas, propositalmente, a condições consideradas satisfatórias para a germinação (Nacimento \& Nascimento, 2021). Esse fenômeno ocorre para que as sementes possam encontrar condições favoráveis para o desenvolvimento da plântula, pois a germinação tende a ser distribuída ao longo do tempo (Floriano, 2004). Assim, a dormência refere-se a um sistema evolutivo que visa a sobrevivência da espécie garantindo longevidade das sementes (Mori et al., 2012).

Para viveiristas, a dormência pode ser notada na prática quando sementes são colocadas em condições favoráveis e não germinam. Este se torna um dos principais problemas para a produção de mudas em viveiros, pois cada espécie possui um tipo específico de dormência e precisa de diferentes condições ambientais para ter essa dormência superada (Brancalion et al., 2015). Antes de escolher o método para quebrar a dormência de sementes é preciso entender o que bloqueia a geminação, já que para cada forma de dormência em sementes existe uma ou mais formas mais adequadas e eficientes (Zaidan \& Barbedo, 2004; Soltoni, et al., 2018).

A dormência de sementes parece possuir alguma associação entre tamanho e permeabilidade de seus tegumentos, principalmente em leguminosas e sementes menores que podem apresentar maior impermeabilidade, sendo relacionada às condições ambientais de desenvolvimento da planta mãe (Nimer et al., 1983; Renzi, 2020). Esse tipo de dormência, imposta pelo tegumento, ocorre quando os envoltórios da semente atuam como uma barreira física que não é superada pelo embrião. Desta forma, o conhecimento dos meios de reversão dos efeitos dessa dormência, além de ser importante economicamente para a produção de mudas, ainda é fundamental para compreender a dinâmica dos bancos de sementes no solo e dos eventos de regeneração em comunidades naturais (Perez, 2004).

Diante dessas informações, o presente trabalho tem como objetivo verificar as características biométricas de sementes de diferentes matrizes de $P$. dubium e relacioná-las ao potencial germinativo e à intensidade de dormência em diferentes tamanhos de semente, visando compreender o comportamento das sementes e facilitar a obtenção de plântulas dessa espécie.

\section{Metodologia}

Este trabalho foi conduzido na Universidade Estadual de Mato Grosso do Sul na Unidade Universitária de Ivinhema. As sementes foram coletadas na zona rural do município, em 12 matrizes que distavam entre si por pelo menos 50 metros (Tabela 1). A circunferência de cada matriz à altura do peito (CAP) foi medida com auxílio de uma fita métrica. As coordenadas das matrizes foram coletadas com um GPS Garmin-Etrex.

Os frutos foram coletados separadamente, com o auxílio do podão, identificado em estágio de frutos maduros (pericarpo com coloração amarronzada), em seguida foram beneficiados manualmente, descartando-se as sementes visualmente danificadas. Os lotes finais para o estudo foram constituídos de 350 sementes por matriz. 
Tabela 1. Coordenadas geográficas e circunferência à altura do peito (CAP) das matrizes de $P$. dubium utilizadas neste estudo.

\begin{tabular}{|c|c|c|c|}
\hline Matriz & \multicolumn{2}{|c|}{ Coordenadas geográficas } & $\mathrm{CAP}(\mathrm{cm})^{*}$ \\
\hline 1 & $22^{\circ} 19^{\prime} 07.33^{\prime \prime} \mathrm{S}$ & $053^{\circ} 50^{\prime} 20.0^{\prime \prime} \mathrm{W}$ & 170 \\
\hline 2 & $22^{\circ} 19^{\prime} 05.6^{\prime \prime} \mathrm{S}$ & $053^{\circ} 50^{\prime} 28.7^{\prime \prime} \mathrm{W}$ & $167+176$ \\
\hline 3 & $22^{\circ} 18^{\prime} 50.7^{\prime \prime} \mathrm{S}$ & $05341^{\prime} 56.1 " \mathrm{~W}$ & $130+70$ \\
\hline 4 & $22^{\circ} 19^{\prime} 19.5^{\prime \prime} \mathrm{S}$ & $053^{\circ} 49^{\prime} 42.0^{\prime \prime} \mathrm{W}$ & $1,55+122$ \\
\hline 5 & $22^{\circ} 17^{\prime} 23.2^{\prime \prime} \mathrm{S}$ & $053^{\circ} 55^{\prime} 47.6^{\prime \prime} \mathrm{W}$ & $193+197$ \\
\hline 6 & $22^{\circ} 19^{\prime} 24.8^{\prime \prime} \mathrm{S}$ & $053^{\circ} 47^{\prime} 59.3^{\prime \prime} \mathrm{W}$ & 132 \\
\hline 7 & $22^{\circ} 19^{\prime} 15.6^{\prime \prime} \mathrm{S}$ & $053^{\circ} 48^{\prime} 27.8^{\prime \prime} \mathrm{W}$ & 221 \\
\hline 8 & $22^{\circ} 21^{\prime} 32.5^{\prime \prime} \mathrm{S}$ & $053^{\circ} 52^{\prime} 09.6^{\prime \prime} \mathrm{W}$ & $1,21+63+1,42+70$ \\
\hline 9 & $22^{\circ} 22^{\prime} 09.4^{\prime \prime} \mathrm{S}$ & $053^{\circ} 54^{\prime} 06.81^{\prime \prime} \mathrm{W}$ & 158 \\
\hline 10 & $22^{\circ} 20^{\prime} 41.7^{\prime \prime} \mathrm{S}$ & $053^{\circ} 52^{\prime} 32.1^{\prime \prime} \mathrm{W}$ & 115 \\
\hline 11 & $22^{\circ} 19^{\prime} 54.8^{\prime \prime} \mathrm{S}$ & $053^{\circ} 54^{\prime} 25.8^{\prime \prime} \mathrm{W}$ & 205 \\
\hline 12 & $22^{\circ} 19^{\prime} 41.2^{\prime \prime} \mathrm{S}$ & $053^{\circ} 50^{\prime} 18.5^{\prime \prime} \mathrm{W}$ & 152 \\
\hline
\end{tabular}

*As circunferências que apresentam mais de uma medida representam matrizes com ramificações. Fonte: Autores (2021).

\section{Biometria}

Cem sementes de cada lote foram separadas aleatoriamente, seguida por análise de sementes biometricamente, foi determinado o comprimento $(\mathrm{mm})$, largura $(\mathrm{mm})$, espessura $(\mathrm{mm})$ e massa fresca $(\mathrm{g})$, com o auxílio de um paquímetro digital $(0,01 \mathrm{~mm})$ e uma balança de precisão $(0,0001 \mathrm{~g})$. O comprimento foi determinado pela medida do eixo maior da semente, da base ao ápice, e a largura e espessura foram obtidas na região mediana da semente. Estes dados foram utilizados para a caracterização das matrizes quanto às dimensões médias, máximas e mínimas das sementes produzidas.

Os dados de biometria reunidos foram submetidos à análise estatística descritiva e à análise de variância (ANOVA um critério), seguida de teste de Tukey a $5 \%$ de probabilidade. O teste de correlação de Pearson, para o mesmo conjunto de dados, foi utilizado para verificação de relação entre as variáveis. Desta forma, foram definidas classes de tamanho das sementes com base na variável massa (pequenas, médias e grandes), a partir de um histograma de distribuição de frequência das sementes de P. dubium.

\section{Germinação}

\section{Comparação das matrizes}

O potencial germinativo de cada lote foi avaliado pela disposição de 100 sementes intactas em quatro placas de Petri, sendo 25 sementes em cada placa, contendo duas folhas de papel filtro que foram mantidas umedecidas com água destilada por todo o experimento. As placas foram mantidas em câmara germinadora de Demanda Bioquímica de Oxigênio (D.B.O), a 25º C, sob luz constante com intensidade de 750lux (Wang et al., 2020).

Esse mesmo procedimento foi adotado para sementes de cada matriz submetidas a tratamento para superação de dormência. Para tanto, outras 100 sementes de cada matriz foram imersas em ácido sulfúrico concentrado por 15 minutos (Souza, Junior \& Brancalion, 2016). Em seguida, lavadas em água corrente por 5 minutos para a remoção do excesso de ácido.

A germinação foi observada diariamente durante 28 dias, sendo considerada germinada a semente cuja raiz primária apresentasse pelo menos $2 \mathrm{~mm}$ de comprimento (Brasil, 2009). Os dados de germinação foram lançados em planilhas do Excel e utilizados para os cálculos de frequência relativa, porcentagem acumulada, velocidade média de germinação (Maguire, 1962), tempo médio de germinação (Edmond \& Drapala, 1959) e índice de sincronização. Estes dados foram submetidos ao 
teste $\mathrm{t}$ de Student (dados paramétricos) ou ao teste U de Mann-Whitney (dados não paramétricos) para comparação das sementes intactas com as escarificadas.

\section{Comparação das categorias de sementes}

Finalizada essa etapa, as sementes restantes, 150 de cada matriz, foram reunidas em um único lote e, em seguida, separadas nas categorias definidas com base na análise de correlação para os dados biométricos. Estas sementes foram usadas para a avaliação do potencial germinativo por categoria, conforme já descrito, com escarificação ácida, para verificar se há influência da categoria na intensidade de dormência. Os ensaios foram realizados sob luz constante e com fotoperíodo de 12 horas. As categorias foram comparadas por análises de variância para dados não paramétricos, Kruskal-Wallis, seguidas por teste de Dunn à 5\% de probabilidade. Todas as análises estatísticas foram realizadas com o auxílio do programa Bioestat 5.3 (Ayres et al., 2007).

\section{Resultados e Discussão}

O experimento foi conduzido no Laboratório de Química da Universidade Estadual de Mato Grosso do Sul, unidade universitária de Ivinhema, Mato Grosso do Sul. Por meio do método quantitativo, foram analisados a biometria e o potencial germinativo de 100 sementes de $P$. dubium.

\section{Biometria}

Os valores médios de massa, comprimento, largura e espessura por matriz estão representados na Tabela 2. As matrizes apresentaram-se heterogêneas quanto aos parâmetros biométricos avaliados. As matrizes 6, 7, 8, 10 e 12, por exemplo, se destacaram pela massa das sementes enquanto a matriz 7 destaca-se pelo comprimento, a 8 em largura e a 6 em espessura.

Tabela 2. Dados biométricos médios das amostras por matriz de sementes de $P$. dubium coletadas.

\begin{tabular}{ccccc}
\hline Matriz & Massa $(\mathrm{g})$ & Comprimento $(\mathrm{mm})$ & Largura $(\mathrm{mm})$ & Espessura $(\mathrm{mm})$ \\
\hline 1 & $0,039 \mathrm{~g}$ & $8,6343 \mathrm{f}$ & $4,013 \mathrm{e}$ & $1,6095 \mathrm{e}$ \\
2 & $0,043 \mathrm{f}$ & $8,7611 \mathrm{f}$ & $3,9656 \mathrm{e}$ & $1,6375 \mathrm{e}$ \\
3 & $0,049 \mathrm{~cd}$ & $9,9358 \mathrm{bc}$ & $4,0723 \mathrm{de}$ & $1,7621 \mathrm{~cd}$ \\
4 & $0,0491 \mathrm{~cd}$ & $9,4361 \mathrm{de}$ & $4,1498 \mathrm{~cd}$ & $1,6888 \mathrm{de}$ \\
5 & $0,0508 \mathrm{bc}$ & $9,5889 \mathrm{~d}$ & $4,3894 \mathrm{~b}$ & $1,686 \mathrm{e}$ \\
6 & $0,053 \mathrm{ab}$ & $9,2747 \mathrm{e}$ & $4,1831 \mathrm{~cd}$ & $1,9814 \mathrm{a}$ \\
7 & $0,0563 \mathrm{a}$ & $10,6514 \mathrm{a}$ & $4,1487 \mathrm{~cd}$ & $1,8728 \mathrm{~b}$ \\
8 & $0,0541 \mathrm{ab}$ & $9,8384 \mathrm{c}$ & $4,7858 \mathrm{a}$ & $1,6611 \mathrm{e}$ \\
9 & $0,0469 \mathrm{de}$ & $9,4418 \mathrm{de}$ & $4,2437 \mathrm{c}$ & $1,6191 \mathrm{e}$ \\
10 & $0,053 \mathrm{ab}$ & $9,9854 \mathrm{bc}$ & $4,4599 \mathrm{~b}$ & $1,7604 \mathrm{~cd}$ \\
11 & $0,0444 \mathrm{ef}$ & $9,5164 \mathrm{de}$ & $3,5923 \mathrm{f}$ & $1,7856 \mathrm{c}$ \\
\hline
\end{tabular}

*Médias acompanhadas por letras iguais não diferem entre si a 5\% de probabilidade. Fonte: Autores (2021).

A análise dos valores máximos e mínimos (Tabela 3) mostraram que há variações em cada matriz para um mesmo parâmetro, evidenciando que uma matriz não produz apenas sementes pequenas, médias ou grandes. As matrizes 1 , 2 e 11 não produziram sementes grandes e a matriz 7 não produziu sementes pequenas. 
Tabela 3. Dados biométricos (máximos e mínimas) das amostras de sementes por matriz.

\begin{tabular}{ccccccccc}
\hline & \multicolumn{2}{c}{ Massa $(\mathrm{g})$} & \multicolumn{3}{c}{ Comprimento $(\mathrm{mm})$} & \multicolumn{2}{c}{ Largura $(\mathrm{mm})$} & \multicolumn{2}{c}{ Espessura $(\mathrm{mm})$} \\
\cline { 2 - 8 } Matriz & Mínimo & Máximo & Mínimo & Máximo & Mínimo & Máximo & Mínimo & Máximo \\
\hline 1 & 0,022 & 0,054 & 7,61 & 9,67 & 2,98 & 4,68 & 1,09 & 1,98 \\
2 & 0,030 & 0,055 & 7,69 & 9,85 & 3,53 & 4,39 & 1,19 & 2,86 \\
3 & 0,029 & 0,065 & 8,93 & 10,99 & 1,14 & 4,56 & 1,44 & 2,09 \\
4 & 0,031 & 0,067 & 8,19 & 10,3 & 3,59 & 4,76 & 1,12 & 2,07 \\
5 & 0,028 & 0,072 & 7,54 & 10,66 & 3,66 & 5,18 & 1,11 & 2,1 \\
6 & 0,031 & 0,074 & 4,75 & 10,4 & 2,22 & 4,83 & 1,42 & 2,44 \\
7 & 0,041 & 0,069 & 9,78 & 11,38 & 3,54 & 4,63 & 1,56 & 2,24 \\
8 & 0,033 & 0,065 & 8,24 & 10,89 & 4,06 & 5,37 & 1,37 & 1,85 \\
9 & 0,030 & 0,072 & 7,5 & 10,47 & 3,39 & 5,08 & 1,05 & 2,11 \\
10 & 0,032 & 0,070 & 8,27 & 11 & 3,81 & 5,12 & 1,29 & 1,97 \\
11 & 0,022 & 0,055 & 8,36 & 10,74 & 1,64 & 4,11 & 1,23 & 2,1 \\
12 & 0,037 & 0,066 & 9,11 & 11,34 & 3,71 & 4,96 & 1,46 & 2,07 \\
\hline
\end{tabular}

Fonte: Autores (2021).

Para Fontenele et al. (2007), estas variações por matriz são importantes, pois possibilitam avaliar a variabilidade genética dos caracteres investigados, buscando a manutenção de áreas com populações com alta diversidade genética. Por outro lado, a influência das condições ambientais geralmente resulta em alterações no tamanho, fisiologia e sanidade das sementes (Macedo et al., 2009). Para Leishman et al. (2000), os inconstantes tamanhos das sementes não são causados por variações genéticas, mas sim por estímulos ambientais durante seu desenvolvimento.

O polimorfismo pode ocorrer dentro de uma mesma matriz, devido à necessidade de dispersão que favorece sementes pequenas e as necessidades de estabelecimento das mudas que favorecem sementes grandes (Malavasi \& Malavasi, 2001). Segundo Freire et al. (2015), mesmo dentro de populações de uma mesma espécie, o tamanho médio das sementes pode variar dependendo das perturbações do meio e do estágio da sucessão vegetal.

As sementes de P. dubium apresentaram em média massa entre 0,039 e 0,056g, comprimento entre 8.6343 e 10.6514 mm, largura variando de 3.5923 a 4.7858 mm e espessura entre 1.6095 e 1.9814 mm (Tabela 4). Os resultados observados para massa (Tabela 4), comprimento, largura e espessura das sementes são semelhantes ao estudo de Andrade (2013), que analisou lotes de sementes da mesma espécie, P. dubium e observou para essas mesmas medias valores de 0.0496g, 9,699mm, 4,211 mm e 1,818mm, respectivamente. A variação exibida na massa de sementes, por exemplo, pode estar relacionada a vários fatores, dentre eles é importante enfatizar a variabilidade genética entre matrizes da mesma espécie, a idade, o estado nutricional e as condições ambientais em que cada árvore está submedida (Müller et al., 2016).

Tabela 4. Estatística descritiva para os dados biométricos médios das sementes de P. dubium.

\begin{tabular}{lcccc}
\hline & Massa $(\mathrm{g})$ & Comprimento $(\mathrm{mm})$ & Largura $(\mathrm{mm})$ & Espessura $(\mathrm{mm})$ \\
\hline Média Aritmética & 0,0495 & 9,5907 & 4,2002 & 1,7273 \\
Mínimo & 0,039 & 8,6343 & 3,5923 & 1,6095 \\
Máximo & 0,0563 & 10,6514 & 4,7858 & 1,9814 \\
Variância & 0 & 0,3172 & 0,0881 & 0,0124 \\
Desvio Padrão & 0,0053 & 0,5632 & 0,2968 & 0,1115 \\
Erro Padrão & 0,0015 & 0,1626 & 0,0857 & 0,0322 \\
Coef, Variação & $10,69 \%$ & $5,87 \%$ & $7,07 \%$ & $6,46 \%$ \\
\hline
\end{tabular}

Fonte: Autores (2021). 
A correlação dos dados biométricos mostrou-se positivamente significativa para todas as comparações, com valores de $r$ mais próximos a 1 (associação mais forte) para as associações com a variável massa (Figura 1).

Figura 1. Correlação de Pearson entre as variáveis biométricas das sementes de $P$. dubium com os respectivos valores de $p$ e $r$.
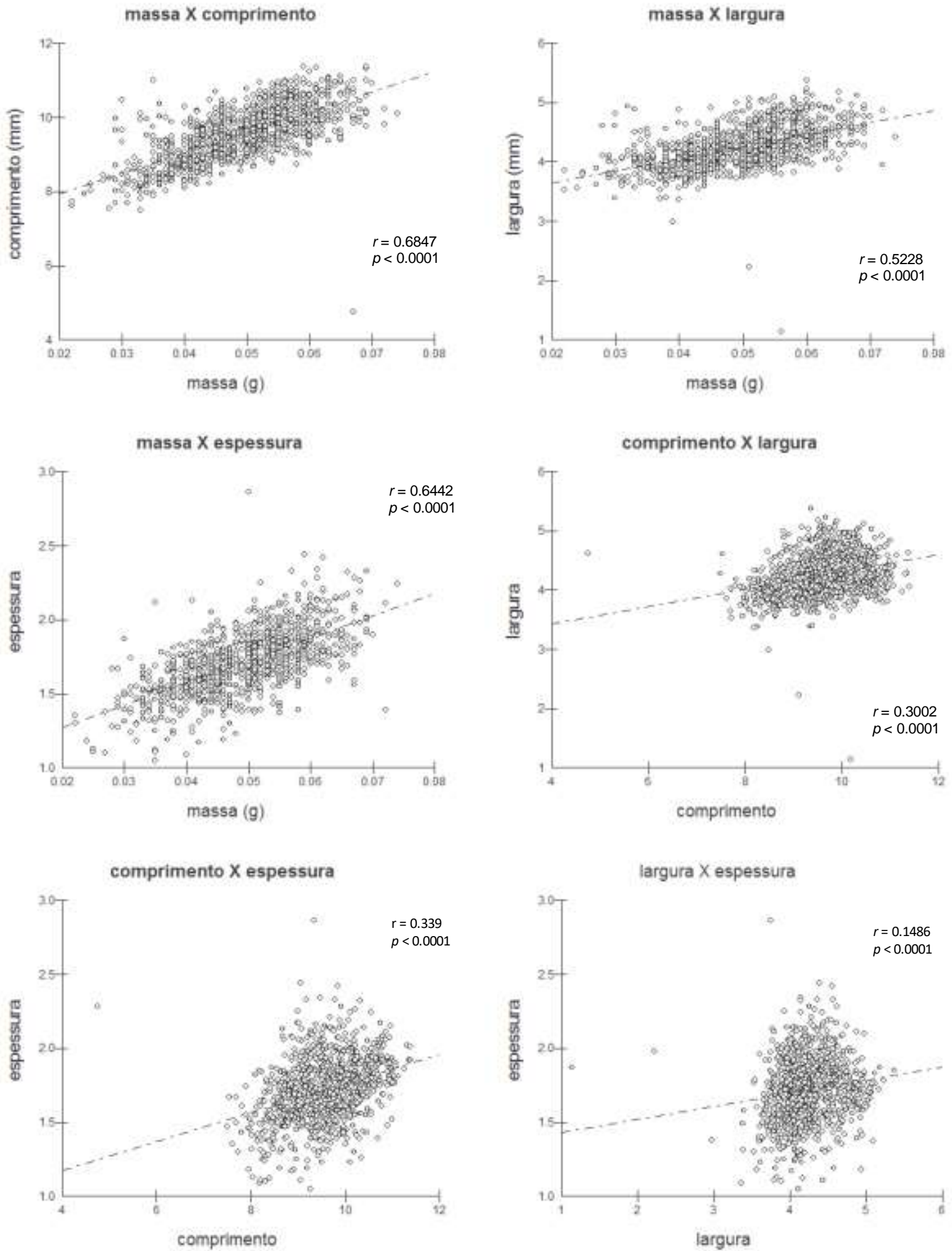

Fonte: Autores (2021). 
Assim, com base na distribuição de frequência das massas das sementes de $P$. dubium, foram consideradas pequenas aquelas com $0,022 \mathrm{~g}$ a $0,039 \mathrm{~g}$, médias as com $0,040 \mathrm{~g}$ a $0,057 \mathrm{~g}$, e grandes as sementes entre $0,058 \mathrm{~g}$ a $0,076 \mathrm{~g}$ (Figura 2).

A seleção de sementes por tamanho vem sendo bastante aplicada para encontrar a categoria ideal para a germinação de cada espécie (Galindo, 2006). O tamanho da semente é uma característica física, afirma Wood et al. (1997) (apud Galindo, 2006) e é determinado pelo genótipo e influências ambientais que atuam durante sua formação. Alves et al. (2005) concluíram que o vigor de sementes possui relação direta com o seu tamanho.

7

Figura 2. Histograma de distribuição da frequência de sementes de $P$. dubium em função das classes de massa.

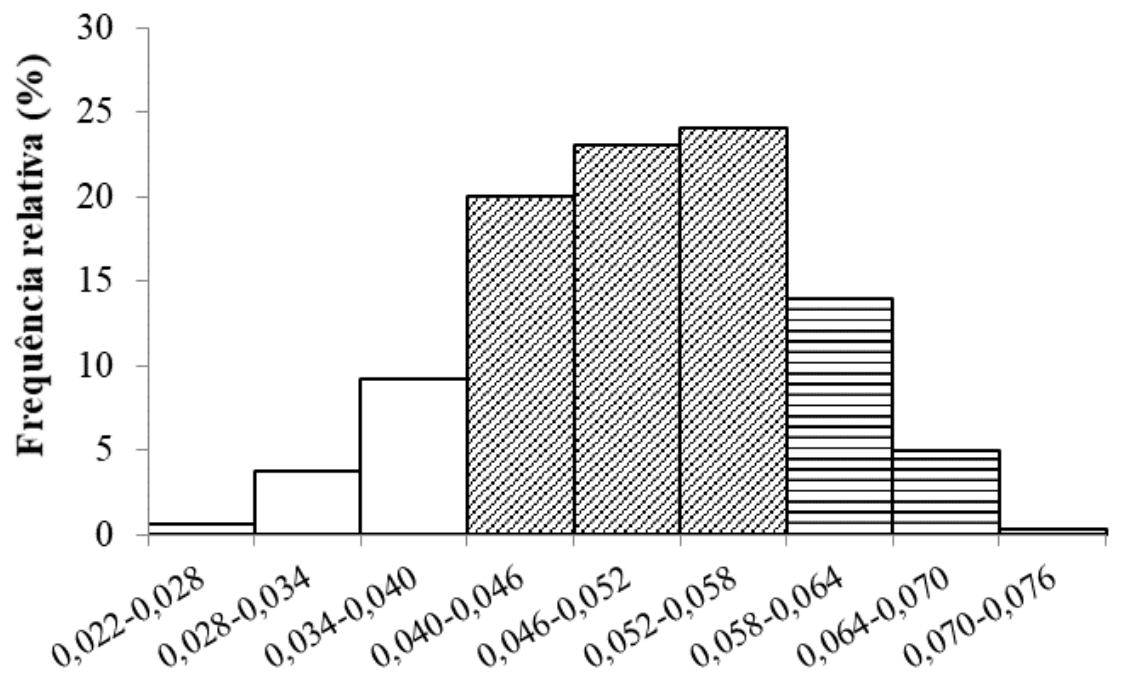

Classes de massa (g)

Fonte: Autores (2021).

\section{Germinação}

Na avaliação por matriz, a germinação de sementes escarificadas foi alta (78\% a 97\%) e baixa (1\% a 11\%) ou nula para as intactas (Tabela 5). O tempo médio de germinação variou de 4.196 a 8,949 dias para as sementes com escarificação química e de 6 a 16 dias para as intactas.

Tabela 5. Porcentagem média final (\% G), tempo médio (Tm), velocidade média (Vm) e índice de sincronização (IS) para a germinação das sementes de $P$. dubium separadas por matriz e submetidas ou não à escarificação química.

\begin{tabular}{ccccc|cccc}
\hline \multirow{2}{*}{ Matriz } & \multicolumn{4}{c|}{ Escarificadas } & \multicolumn{4}{c}{ Intactas } \\
\cline { 2 - 9 } & $\% \mathrm{G}$ & $\begin{array}{c}\text { Tm } \\
(\text { dias })\end{array}$ & $\begin{array}{c}\text { Vm } \\
\left(\text { dias }^{-1}\right)\end{array}$ & IS (bits) & $\%$ G & $\begin{array}{c}\text { Tm } \\
(\text { dias })\end{array}$ & $\begin{array}{c}\text { Vm } \\
\left(\text { dias }^{-1}\right)\end{array}$ & $\begin{array}{c}\text { IS } \\
(\text { bits })^{2}\end{array}$ \\
\hline 1 & 83 & 5,289 & 0,189 & 2,724 & 0 & 0 & 0 & 0 \\
2 & 90 & 6,933 & 0,144 & 3,758 & 2 & 11 & 0,091 & 0 \\
3 & 89 & 4,932 & 0,203 & 1,122 & 4 & 6,5 & 0,154 & 2 \\
4 & 97 & 4.196 & 0.238 & 1.031 & 3 & 5,667 & 0,176 & 0,918 \\
5 & 94 & 5,691 & 0,176 & 5,238 & 9 & 7,444 & 0,134 & 1,98 \\
6 & 87 & 4,931 & 0,203 & 3,125 & 11 & 6,182 & 0,162 & 1,972 \\
7 & 96 & 4,698 & 0,213 & 2,704 & 3 & 7 & 0,143 & 1,585 \\
8 & 97 & 4,423 & 0,226 & 1,709 & 0 & 0 & 0 & 0 \\
9 & 88 & 5,204 & 0,192 & 4,296 & 9 & 11,222 & 0,089 & 2,818 \\
10 & 84 & 6,036 & 0,166 & 5,762 & 5 & 8,6 & 0,116 & 1,371 \\
11 & 78 & 8,949 & 0,112 & 3,725 & 1 & 16 & 0,062 & 0 \\
12 & 97 & 4,175 & 0,239 & 0,601 & 1 & 6 & 0,167 & 0 \\
\hline
\end{tabular}

Fonte: Autores (2021). 
Alta porcentagem germinativa indica que o tratamento aplicado possibilitou a embebição, não comprometendo o embrião, e elevados valores de velocidade média indicam que a germinação ocorreu em um curto período (Perez, 2004). Estes dados mostram como é útil a escarificação quando o objetivo é homogeneizar a germinação de sementes com dormência física e estão de acordo com Souza, Junior e Brancalion (2016), que relataram a germinação esperada de $P$. dubium sendo de 80 a $100 \%$ em até 15 dias.

As médias gerais para porcentagem, tempo médio e velocidade média de germinação foram, respectivamente, $90 \%$, 5,454 dias e 0,1523 dias $^{-1}$ para as sementes escarificadas, e 4\%, 7,134 dias e 0,108 dias ${ }^{-1}$ para as intactas (Tabela 6).

Tabela 6. Comparação do tratamento de escarificação química na germinação de sementes de $P$. dubium em relação ao controle (sementes intactas) quanto à porcentagem média final (\% G), tempo médio (Tm), velocidade média (Vm) e índice de sincronização (IS).

\begin{tabular}{|c|c|c|c|c|c|}
\hline Tratamento & & $\% \mathrm{G}$ & $\mathrm{Tm}$ & $\operatorname{Vm}\left(\operatorname{dias}^{-1}\right)$ & IS (bits) \\
\hline Escarificadas & & 90 & 5,454 & 0,1523 & 2,983 \\
\hline Intactas & & 4 & 7,135 & 0,108 & 1,054 \\
\hline & $P$ & $<0,0001$ & 0,0496 & 0,0005 & 0,0026 \\
\hline & Valor do teste & $t=40,4050$ & $\mathrm{U}=38.00$ & $t=4.0424$ & $\mathrm{t}=3,3914$ \\
\hline
\end{tabular}

Fonte: Autores (2021).

Em outro estudo com a mesma espécie, Piroli et al. (2005) descreveram que o tratamento das sementes com ácido sulfúrico, com o mesmo tempo de imersão, obteve a segunda maior porcentagem germinativa $83 \%$, sendo que a primeira, de 89\%, semelhante ao valor obtido neste estudo, foi resultante da escarificação mecânica manual com lixa. Mas esta forma de escarificação mecânica se torna inviável para aplicação em larga escala, principalmente quando as sementes são pequenas, como as de P. dubium. Essa diferença, segundo Perez (2004), pode ocorrer pela dificuldade em escarificar todas as sementes homogeneamente permitindo a permanência de sementes impermeáveis à água ou danificadas.

A sincronia (IS), índice relacionado à frequência relativa de germinação (Figura 3), foi maior em sementes intactas (Tabela 5), o que pode ser explicado pela baixa germinação (nenhum ou poucos picos) no período avaliado (28 dias) em comparação com as sementes que foram escarificadas.

A distribuição da frequência relativa no tempo (Figura 3) permitiu visualizar a rapidez do processo germinativo nas sementes escarificadas, com o principal pico ocorrendo no quarto dia. Para as sementes intactas, a pronta germinação de algumas sementes nos dias iniciais dos ensaios pode ser relacionada à existência de fissuras no tegumento ou a diferentes graus de dormência no lote. A maioria delas, no entanto, não germinaram no tempo avaliado. Para Perez (2004), a distribuição da germinação no tempo é uma característica de sementes dormentes que permite que a germinação inicie quando as condições ambientais estiverem favoráveis.

Figura 3. Frequência relativa e tempo de germinação das sementes intactas e escarificadas por matriz.
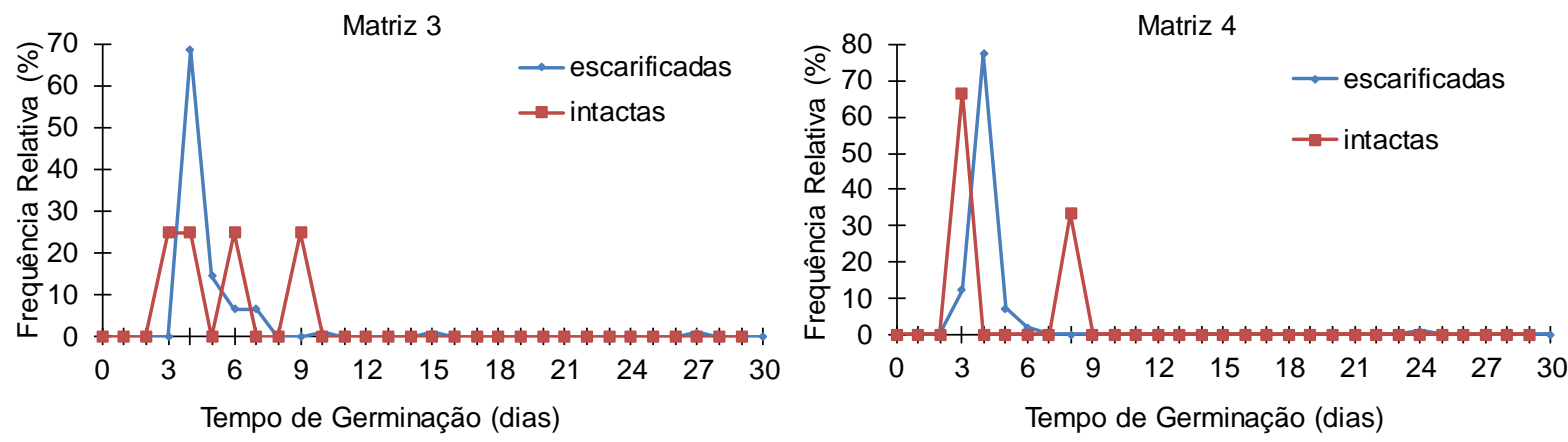


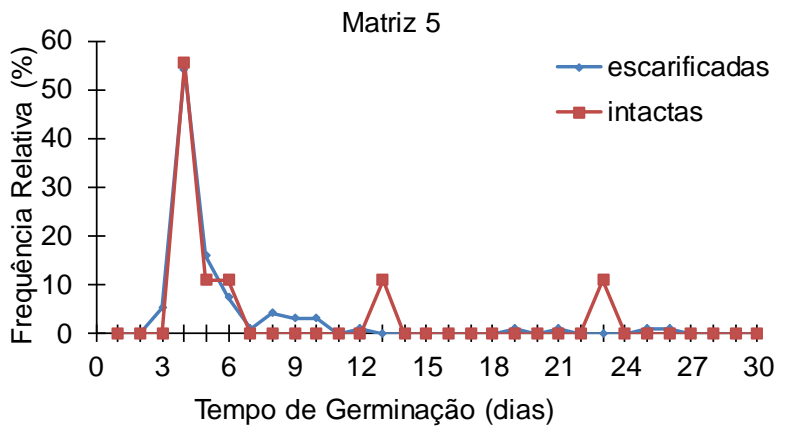

Matriz 7

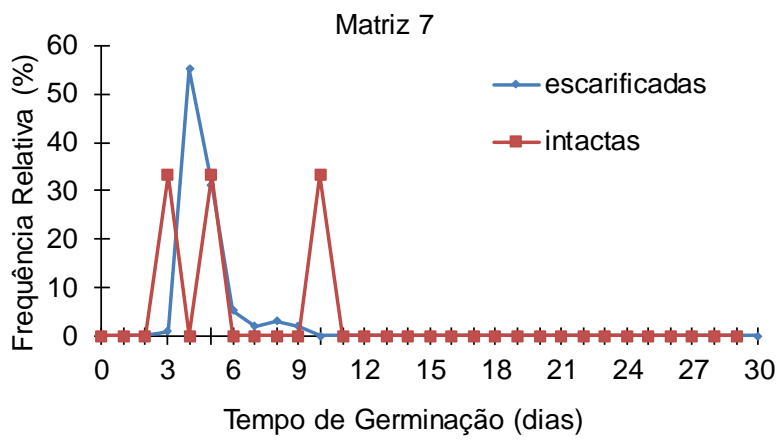

Matriz 9
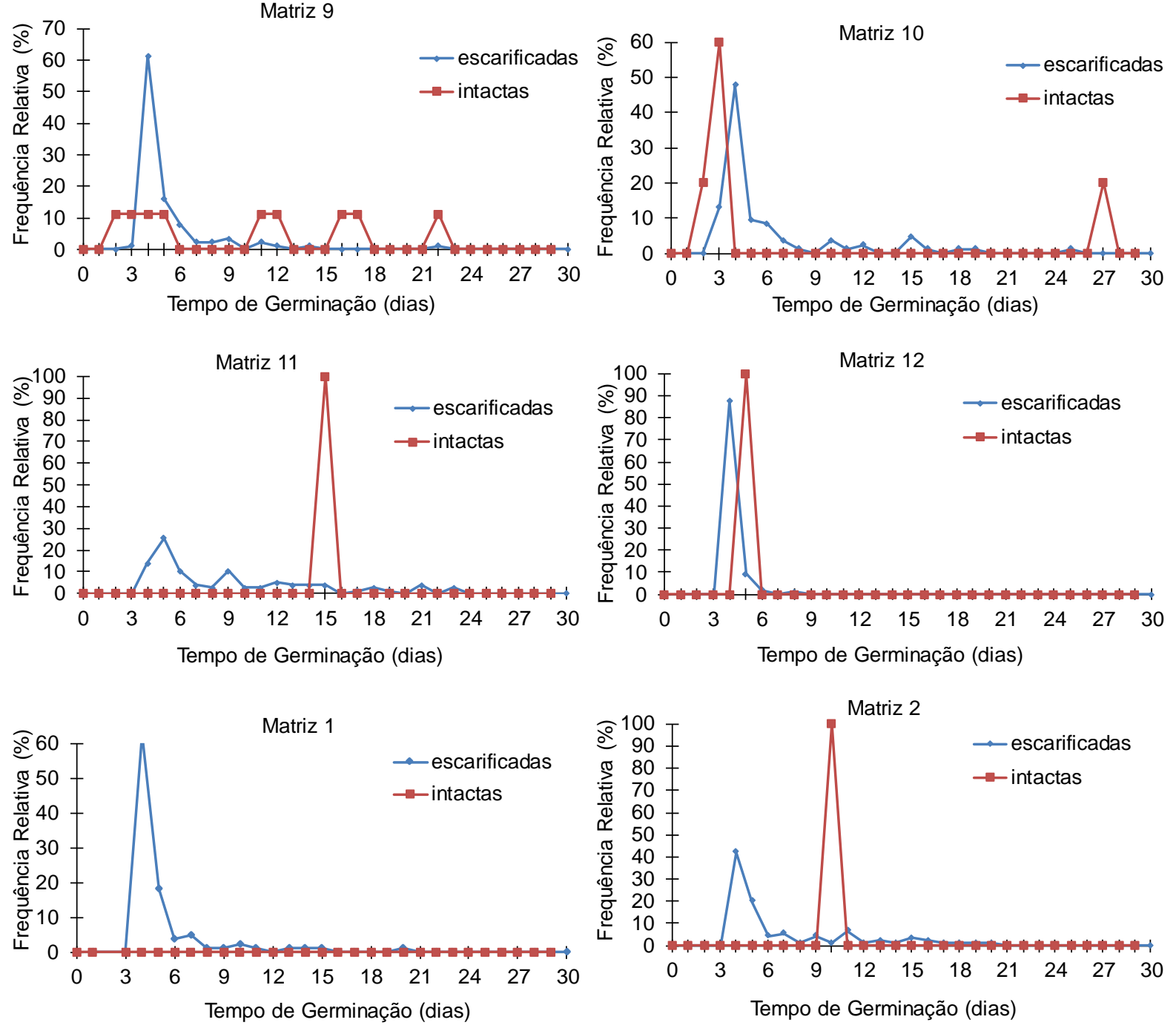

Fonte: Autores (2021). 
A diferença no potencial germinativo pode ser verificada ainda entre as categorias de sementes, tendo as classes de massa médias e grandes apresentado melhor desempenho $($ H12horas $=9,1354, \mathrm{p}=0,0104$; luz contínua = 9.152, $\mathrm{p}=0,0103)$ (Tabela 7). A condição de iluminação (12 horas de luz e luz contínua) não influenciou este resultado $(\mathrm{t}=-0,0932, \mathrm{p}=0,9266)$.

As menores porcentagens germinativas observadas para as sementes pequenas, mesmo depois de imersas em ácido sulfúrico, provavelmente estão relacionadas à menor permeabilidade do tegumento destas em comparação às sementes grandes (Galindo, 2006).

Tabela 7. Porcentagem média final (\% G), tempo médio (Tm), velocidade média (Vm) e índice de sincronização (IS) para a germinação das sementes de $P$. dubium separadas por classes de tamanho e expostas a diferentes condições de iluminação, 12 horas de luz ou luz contínua.

\begin{tabular}{cccccc}
\hline \multicolumn{2}{l}{ Tamanho da Semente } & $\% \mathrm{G}^{*}$ & $\mathrm{Tm}(\mathrm{dias})$ & $\mathrm{Vm}\left(\mathrm{dias}^{-1}\right)$ & IS (bits) \\
\hline $\mathrm{P}$ & \multirow{2}{*}{ 12 horas } & $84 \mathrm{bA}$ & 4,012 & 0,249 & 1,079 \\
$\mathrm{M}$ & & $96 \mathrm{abA}$ & 3,729 & 0,268 & 1,007 \\
$\mathrm{G}$ & $100 \mathrm{aA}$ & 3,680 & 0,272 & 1,112 \\
\hline $\mathrm{P}$ & & $83 \mathrm{bA}$ & 4,578 & 0,218 & 0,615 \\
$\mathrm{M}$ & Luz contínua & $100 \mathrm{aA}$ & 4,000 & 0,250 & 0,379 \\
$\mathrm{G}$ & & $98 \mathrm{abA}$ & 3,990 & 0,251 & 0,338 \\
\hline
\end{tabular}

*Médias acompanhadas por letras iguais não diferem entre si a 5\% de probabilidade. As letras minúsculas referem-se à comparação dentro de um mesmo fotoperíodo e as maiúsculas comparam tamanhos de sementes em fotoperíodos diferentes. Fonte: Autores (2021).

\section{Conclusão}

Após às análises realizadas no presente estudo, podemos inferir que a germinabilidade dos lotes de sementes de $P$. dubium após escarificação foi alta (acima de 78\%), sendo que a biometria apresentou dados semelhantes aos esperados para a região. Considerando a variabilidade observada entre as matrizes encontradas no município como sendo um importante fator, a destacamos como característica a ser mantida nesta população. Concluímos que a massa pode ser utilizada para a seleção de sementes desta espécie e que as sementes médias e grandes apresentaram maior potencial germinativo.

Destacamos a importância dos resultados obtidos neste estudo, já que que estes podem auxiliar viveiristas na seleção de sementes para a germinação e fornecer informações ecologicamente relevantes sobre os processos de quebra de dormência de P. dubium. Assim, salientamos que a realização de novos estudos envolvendo aspectos biológicos como os apresentados no presente trabalho, são fundamentais para compor um acervo de informações que podem contribuir de maneira significativa no entendimento dos processos de quebra de dormência e germinação de sementes.

\section{Referências}

Alves, E. U., Bruno, R. L. A., Oliveira, A. P., Alves, A. U., Alves, A. U. \& Paula, R. C. (2005). Influência do Tamanho e da procedência de Sementes de Mimosa caesalpiniifolia Benth. Sobre a Germinação e Vigor. Revista Árvore, 6, 877-885.

Andrade, L. D. (2013). Características Biométricas das Sementes e Descrição da Resposta Germinativa pelo Modelo do Tempo Térmico em Diferentes Populações De Peltophorum dubium (Spreng.) Taub. Trabalho de Dissertação - Instituto de Biociências da Universidade Estadual Paulista "Júlio Mesquita Filho", Rio Claro, SP, p. 21-32.

Ayres, M., Ayres Junior, M., Ayres, D. L. \& Santos, A. A. (2007). Bioestat aplicações estatísticas nas áreas das ciências biomédicas. Mamiraua. Belém, PA.

Brancalion, P. H. S. \& Gandolfi, S., Rodrigues, R. R. (2015). Produção de mudas de espécies nativas para fins de restauração. In: Restauração Florestal. Brancalion, P.H.S; Gandolfi, S.; Rodrigues, R.R. Oficina de textos, p. 356.

Brasil (2009). Ministério da Agricultura, Pecuária e Abastecimento. RAS - Regra Gerais para Análise de sementes. $147-224$.

Carvalho, N. M. \& Nakagawa, J. (2000). Sementes: ciência, tecnologia e produção, 4.ed. Jaboticabal: FUNEP.

Cruz, E. D., Martins, F. O. \& Carvalho, J. E. U. (2001). Biometria de frutos e sementes e germinação o de jatobá-curuba (Hymenaea intermedia Ducke, Leguminosae - Caesalpinioideae). Revista brasileira de Botânica, São Paulo, 24 (2), 161-165. 
Edmond, R. \& Drapala, W. J. (1959). The effect of temperature, imersion ia acetone and sulfuric acid on germination of five varieties of okra seed. Proceedings of the American Society for Horticultural Science, St. Joseph, 74, 60I-606.

Ermínio, I. B., Martins, K. V., Bitar, N. A. B. \& Dias, A. A. V. (2016). Superação de Dormência de Sementes de Canafítula [Peltophorum dubium (Sprengel) Taubert]. Revista Cerrado Agrociências. Patos de Minas, 7, 58-65.

Fenner, M. (1993). Seed ecology. Chapman \& Hall, London.

Floriano, E. P. (2004). Germinação e Dormência de Sementes Florestais. http://files.engflorestal.webnode.com.br/2000000 1272bd573b79/Germina\%C3\%A7\%C3\%A3o\%20e\%20Dorm\%C3\%AAncia\%20de\%20sementes\%20florestais.pdf.

Fontenele, A. C. F., Aragão, W. M. \& Rangel, J. H. A. (2007). Biometria de Frutos e Sementes de Desmanthus virgatus (L) Willd Nativas de Sergipe. Revista Brasileira de Biociências, Porto Alegre, 5(1), 252-254.

Freire, J. M., Piña-Rodrigues, F. C. M., Santos, A. L. F. \& Pereira, M. B. (2015). Variação Entre e Dentro de Populações em Tamanho e Dormência de Sementes de Schizolobium parahyba (Vell.) Blake Na Floresta Atlântica. Ciência Florestal, 25(4).

Galindo, C. A. M. (2006). Absorção de Água, Germinação e Dormência de Sementes de Mucuna Preta. Dissertação (Mestrado em Ciências Agrárias e Veterinária) - Unesp, Campus de Jaboticabal, SP.

Gusmão, E.; Vieira, F.A. \& Júnior, E. M. F. (2006). Biometria de frutos e endocarpos de murici (Byrsonima verbascifolia Rich. ex A. Juss.). Revista Cerne, Lavras, 12(1), 84-91.

Leishman, M. R., Wright, I. J., Moles, A.T. \& Westoby, M. (2000). The evolutionary ecology of seed size. Pp. 31-57. In: Fenner, M (ed.). Seeds - the ecology of regeneration in plant communities. Wallingford, CAB International.

Lorenzi, H. (1992). Árvores brasileiras: manual de identificação e cultivo de plantas arbóreas nativas do Brasil. Nova Odessa, SP: Editora Plantarum.

Macedo, M. C., Scalon, S. P. Q., Sari, A. P., Scalon Filho, H., Rosa, Y. B. C. J. \& Robaina, A. D. (2009). Biometria de frutos e sementes e germinação de Magonia pubescens ST. Hil (SAPINDACEAE). Revista Brasileira de Sementes, 31(2), $202-211$.

Maguire, J. D. (1962). Speed of germination aid in selection and evaluation for seedling emergence and vigor. Crop Science, Madison, 2(2), $176-77$.

Malavasi, U. C. \& Malavasi, M. M. (2001). Influência do Tamanho e do Peso da Semente na Germinação e no Estabelecimento de Espécies de Diferentes Estágios de Sucessão vegetal. Revista Floresta e Ambiente, 8(1), 211-215.

Mori, E.S., Pina-Rodrigues, F. C. M. \& Freitas, N. P. (2012). Sementes florestais: guia para a germinação de 100 espécies nativas. São Paulo: Instituto Refloresta, p. 19-97.

Müller, E. M., Gibbert, P., Binotto, T., Kaiser, D. K. \& BortolinI, M. F. (2016). Maturação e dormência em sementes de Peltophorum dubium (Spreng) Taub. de diferentes árvores matrizes. Revista Iheringia, Série Botânica, Porto Alegre.

Nascimento, V. E. \& Nascimento, S.F (202. Superação de dormência em sementes da Cassia grandis L. f. (Fabaceae). Revista Verde 16(1), 89-96

Nimer, R., Carvalho, N. M., Loureiro, N. \& Perecini, D. (1983). Influência de alguns fatores da planta sobre o grau de dormência em sementes de mucunapreta. Revista Brasileira de Sementes, ABRATES, Brasília, 5(2), 111-119.

Perez, S. C. J. G. A. (2004). Envoltórios. In: Ferreira, A. G.; Borghetti, F. (Orgs.). Germinação: do básico ao aplicado. Porto Alegre: Artmed, p. 125-134.

Pilori, E. L., Custódio, C. C., Rocha, M. R. V. \& Udenal, J.L. (2005). Germinação de sementes de canafítula Peltophorum dubium (Spreng.) Taub. tratadas para superação de dormência. Revista Colloquium Agrariae, 1(1), 13-18.

Renzi, J. P., Duchoslav, M., Brus, J., Hradilová, I., Pechanec, V., Václavek, T. \& Smýkal, P. (2020). Physical Dormancy Release in Medicago truncatula Seeds Is Related to Environmental Variations. Plants, 9(4), 503.

Santos, F. S. (2007). Biometria, germinação e qualidade fisiológica de sementes de Tabebuia chrysotricha (Mart. ex A. DC.) Standl. provenientes de diferentes matrizes. Dissertação (Mestrado em Agronomia (Produção e Tecnologia de Sementes). Unesp, Câmpus de Jaboticabal, SP. UNESP.

Santos, F. S., Paula, R. C., Sabonaro, D. Z. \& Valadares, J. (2009) Biometria e qualidade fisiológica de sementes de diferentes matrizes de Tabebuia chrysotricha (Mart. Ex A. DC.) StandI. Scientia Forestalis, 37(82), 163-173.

Soltani, E., Baskin, C. C., Baskin, J. M., Heshmati, S. \& Mirfazeli, M. S. (2018). A meta-analysis of the effects of frugivory (endozoochory) on seed germination: role of seed size and kind of dormancy. Plant Ecology, 219(11), 1283-1294.

Souza Junior, C. N. \& Brancalion, P. H. S. (2016). Sementes e Mudas: guia para propagação de árvores brasileiras. 1. ed. São Paulo: Oficina Textos.

Wang, S., Cheng, H., Wei, M., Wu, B. \&Wang, C. (2020). Litter decomposition process dramatically declines the allelopathy of Solidago canadensis L. on the seed germination and seedling growth of Lactuca sativa L. International Journal of Phytoremediation, p. 1-9.

Zaidan, L. B. P. \& Barbedo, C. J. (2004). Quebra de dormência. In: Ferreira, A. G; Borghetti, F. (orgs.). Germinação: do básico ao aplicado. Porto Alegre: Artmed. 